\title{
A generalized form of Grüss type inequality and other integral inequalities
}

\author{
Nicuşor Minculete ${ }^{1 *}$ and Loredana Ciurdariu ${ }^{2}$
}

\author{
${ }^{\text {"Correspondence: }}$ \\ minculeten@yahoo.com \\ ${ }^{1}$ Transilvania University of Brasov, \\ Iuliu Maniu 50, Brasov, 500091 , \\ Romania \\ Full list of author information is \\ available at the end of the article
}

\begin{abstract}
The aim of this presentation is to show several integral inequalities. Among these inequalities we have the inequality $\operatorname{var}_{h}(f) \leq\left(\Gamma_{1}-M_{h}[f]\right)\left(M_{h}[f]-\gamma_{1}\right)$, where $\operatorname{var}_{h}(f)$ denotes the $h$-variance of $f$, which is a bounded function defined on $[a, b]$ with $\gamma_{1} \leq f(x) \leq \Gamma_{1}$, and $\gamma_{1}, \Gamma_{1}$ are two constants. This inequality is important because it proves a generalized form of the Grüss type inequality. This improvement is given by the inequality

$$
0 \leq \frac{\left[\operatorname{cov}_{h}(f, g) \operatorname{cov}_{h}(f, q)-\operatorname{cov}_{h}(g, q) \operatorname{var}_{h}(f)\right]^{2}}{\operatorname{var}_{h}(f) \operatorname{var}_{h}(q)-\left[\operatorname{cov}_{h}(f, g)\right]^{2}} \leq \operatorname{var}_{h}(f) \operatorname{var}_{h}(g)-\left[\operatorname{cov}_{h}(f, g)\right]^{2}
$$

Using the integral arithmetic mean and $h$-integral arithmetic mean for a Riemann-integrable function $f$ we can also rewrite several integral inequalities. In addition, we will give a generalization of inequality of Grüss for normalized isotonic linear functionals.
\end{abstract}

MSC: 26D15; 26D10

Keywords: Grüss type inequality; $h$-variance; $h$-covariance

\section{Introduction}

In 1935, Grüss [1] proved the following integral inequality which gives an approximation for the integral of a product of two functions in terms of the product of integrals of the two functions:

Let $f$ and $g$ be two bounded functions defined on $[a, b]$ with $\gamma_{1} \leq f(x) \leq \Gamma_{1}$ and $\gamma_{2} \leq$ $g(x) \leq \Gamma_{2}$, where $\gamma_{1}, y_{2}, \Gamma_{1}, \Gamma_{2}$ are four constants. Then we have

$$
\left|\frac{1}{b-a} \int_{a}^{b} f(x) g(x) d x-\frac{1}{b-a} \int_{a}^{b} f(x) d x \frac{1}{b-a} \int_{a}^{b} g(x) d x\right| \leq \frac{1}{4}\left(\Gamma_{1}-\gamma_{1}\right)\left(\Gamma_{2}-\gamma_{2}\right)
$$

and the inequality is sharp, in the sense that the constant $1 / 4$ cannot be replaced by a smaller one.

It is well known that an important resource for studying inequalities is [2-4]. In [5], Peng and Miao established a form of inequality of Grüss type for functions whose first and second derivatives are absolutely continuous and the third derivative is bound. Also, in [6] Dragomir presented several integral inequalities of Grüss type, and in [7] he showed some Grüss type inequalities in inner product spaces and applications for the integral. Another improvement of the Grüss inequality was obtained by Mercer in [8]. Moreover,

\section{Springer}

(02014 Minculete and Ciurdariu; licensee Springer. This is an Open Access article distributed under the terms of the Creative Commons Attribution License (http://creativecommons.org/licenses/by/2.0), which permits unrestricted use, distribution, and reproduction in any medium, provided the original work is properly cited. 
in [9], a Grüss type inequality was used in order to obtain some sharp Ostrowski-Grüss type inequalities by Liu. Kechriniotis and Delibasis showed in [10] several refinements of inequality of Grüss in inner product spaces using a Kurepa's results for Gramians. New generalizations of the inequality of Gruss were presented in [11] using Riemann-Liouville fractional integrals. Cerone and Dragomir studied in [12] some refinements of Grüss' inequality. Florea and Niculescu in [13] treated the problem of estimating the deviation of the values of a function from its mean value.

The estimation of the deviation of a function from its mean value is characterized in terms of random variables.

We denote by $\mathcal{R}([a, b])$ the space of Riemann-integrable functions on the interval $[a, b]$, and by $C^{0}([a, b])$ the space of real-valued continuous functions on the interval $[a, b]$.

The integral arithmetic mean for a Riemann-integrable function $f:[a, b] \rightarrow \mathbf{R}$ is the number

$$
M_{1}[f]=\frac{1}{b-a} \int_{a}^{b} f(x) d x
$$

If $f, h \in \mathcal{R}([a, b])$ and $\int_{a}^{b} h(x) d x>0$, then a generalization for the integral arithmetic mean is the number

$$
M_{h}[f]=\frac{\int_{a}^{b} f(x) h(x) d x}{\int_{a}^{b} h(x) d x}
$$

called the $h$-integral arithmetic mean for a Riemann-integrable function $f$.

We find the following property of the $h$-integral arithmetic mean for a Riemannintegrable function $f$ :

$$
M_{h}[f \pm k]=M_{h}[f] \pm k,
$$

where $k$ is a real constant.

If the function $f$ is a Riemann-integrable function, we denote by

$$
\operatorname{var}(f)=M_{1}\left[\left(f-M_{1}(f)\right)^{2}\right]
$$

the variance of $f$.

The expression for the variance can be expanded thus:

$$
\operatorname{var}(f)=\frac{1}{b-a} \int_{a}^{b}\left(f(x)-\frac{1}{b-a} \int_{a}^{b} f(t) d t\right)^{2} d x
$$

In the same way we defined the $h$-variance of a Riemann-integrable function $f$ by

$$
\operatorname{var}_{h}(f)=M_{h}\left[\left(f-M_{h}(f)\right)^{2}\right]
$$

The expression for the $h$-variance can be expanded thus:

$$
\operatorname{var}_{h}(f)=\frac{1}{\int_{a}^{b} h(x) d x} \int_{a}^{b}\left(f(x)-\frac{\int_{a}^{b} f(t) h(t) d t}{\int_{a}^{b} h(t) d t}\right)^{2} h(x) d x .
$$


It is easy to see another form of the $h$-variance, given by the following:

$$
\operatorname{var}_{h}(f)=M_{h}\left[f^{2}\right]-M_{h}^{2}[f]
$$

We note the following property of the $h$-variance of an integrable function $f$ :

$$
\operatorname{var}_{h}(f \pm k)=\operatorname{var}_{h}(f)
$$

where $k$ is a constant.

In [14], Aldaz showed a refinement of the AM-GM inequality and used in the proof that

$$
\frac{1-\int_{a}^{b} f^{\frac{1}{2}}(x) d x}{\left(\int_{a}^{b} f(x) d x\right)^{\frac{1}{2}}}
$$

is a measure of the dispersion of $f^{\frac{1}{2}}$ about its mean value, which is, in fact, comparable to the variance,

$$
\operatorname{Var}\left(\frac{f^{\frac{1}{2}}(x)}{\left\|f^{\frac{1}{2}}(x)\right\|_{2}}\right)
$$

where $\|f(x)\|_{2}=\sqrt{\int_{a}^{b} f^{2}(x) d x}$.

The covariance is a measure of how much two Riemann-integrable functions change together and is defined as

$$
\operatorname{cov}(f, g)=M_{1}\left[\left(f-M_{1}[f]\right)\left(g-M_{1}[g]\right)\right]
$$

and it is equivalent to the form

$$
\begin{aligned}
\operatorname{cov}(f, g) & =M_{1}[f g]-M_{1}[f] M_{1}[g] \\
& =\frac{1}{b-a} \int_{a}^{b} f(x) g(x) d x-\frac{1}{b-a} \int_{a}^{b} f(x) d x \frac{1}{b-a} \int_{a}^{b} g(x) d x .
\end{aligned}
$$

In fact the covariance is the Chebyshev functional attached to functions $f$ and $g$. In [15] is written as $T(f, g)$. The properties of the Chebyshev functional have been studied by Elezović, Marangunić and Pečarić in their paper, [16]. For other generalizations of the Grüss' inequality, see $[17,18]$.

The $h$-covariance is a measure of how much two integrable functions change together and is defined as

$$
\operatorname{cov}_{h}(f, g)=M_{h}\left[\left(f-M_{h}[f]\right)\left(g-M_{h}[g]\right)\right],
$$

and it is equivalent to the form

$$
\begin{aligned}
\operatorname{cov}_{h}(f, g) & =M_{h}[f g]-M_{h}[f] M_{h}[g] \\
& =\frac{\int_{a}^{b} f(x) g(x) h(x) d x}{\int_{a}^{b} h(x) d x}-\frac{\int_{a}^{b} f(x) h(x) d x}{\int_{a}^{b} h(x) d x} \cdot \frac{\int_{a}^{b} g(x) h(x) d x}{\int_{a}^{b} h(x) d x} .
\end{aligned}
$$


In [18], Pečarić used the generalization of the Chebyshev functional notion attached of functions $f$ and $g$ to the Chebyshev $h$-functional attached of functions $f$ and $g$ defined by $T(f, g ; h)$. Here, Pečarić showed some generalizations of the inequality of Grüss by the Chebyshev $h$-functional. It is easy to see that, in terms of the covariance, this can be written as $T(f, g ; h)=\operatorname{cov}_{h}(f, g)$.

In terms of covariance the inequality of Grüss becomes

$$
|\operatorname{cov}(f, g)| \leq \frac{1}{4}\left(\Gamma_{1}-\gamma_{1}\right)\left(\Gamma_{2}-\gamma_{2}\right)
$$

and in terms of Chebyshev functional the inequality of Grüss becomes

$$
|T(f, g)| \leq \frac{1}{4}\left(\Gamma_{1}-\gamma_{1}\right)\left(\Gamma_{2}-\gamma_{2}\right)
$$

Let $x_{1}, x_{2}, \ldots, x_{n}$ be real numbers, assume $m \leq x_{i} \leq M$ for all $i=\overline{1, n}$ and the average $\bar{x}=$ $\frac{1}{n} \sum_{i=1}^{n} x_{i}$, and $X$ a discrete random variable given by

$$
X=\left(\begin{array}{c}
x_{i} \\
\frac{1}{n}
\end{array}\right)_{i=\overline{1, n}} .
$$

In 1935, Popoviciu [19] proved the following inequality:

$$
\operatorname{Var}(X)=\frac{1}{n} \sum_{i=1}^{n}\left(x_{i}-\bar{x}\right)^{2} \leq \frac{1}{4}(M-m)^{2} .
$$

Bhatia and Davis showed in [20] that the following inequality holds:

$$
\operatorname{Var}(X)=\frac{1}{n} \sum_{i=1}^{n}\left(x_{i}-\bar{x}\right)^{2} \leq(M-\bar{x})(\bar{x}-m)
$$

The inequality of Bhatia and Davis represents an improvement of Popoviciu's inequality, because $(M-m)^{2} \geq 4(M-\bar{x})(\bar{x}-m)$.

If there is additional information about the mean values of the two functions in the inequality of Grüss then Zitikis argued in his paper, [21], that the inequality can be sharpened and he gave also a probabilistic interpretation for it.

\section{Main results}

We will present in this paper the integral version of inequalities (3) and (4). Therefore we have the following inequalities.

Lemma 1 Let $f$ be a Riemann-integrable function defined on $[a, b]$ with $\gamma_{1} \leq f(x) \leq \Gamma_{1}$, where $\gamma_{1}$ and $\Gamma_{1}$ are two constants. Then we have

$$
\operatorname{var}_{h}(f) \leq \frac{1}{4}\left(\Gamma_{1}-\gamma_{1}\right)^{2}
$$

where $h:[a, b] \rightarrow[0, \infty)$ is a Riemann-integrable function with $\int_{a}^{b} h(x) d x>0$. 
Proof Since $\gamma_{1} \leq f(x) \leq \Gamma_{1}$, we obtain the following inequality:

$$
\left|f(x)-\frac{\Gamma_{1}+\gamma_{1}}{2}\right| \leq \frac{\Gamma_{1}-\gamma_{1}}{2}
$$

But it is easy to see that

$$
\begin{aligned}
\operatorname{var}_{h}(f) & =\operatorname{var}_{h}\left(f-\frac{\Gamma_{1}+\gamma_{1}}{2}\right) \\
& =M_{h}\left[\left(f-\frac{\Gamma_{1}+\gamma_{1}}{2}\right)^{2}\right]-M_{h}^{2}\left[f-\frac{\Gamma_{1}+\gamma_{1}}{2}\right] \\
& \leq M_{h}\left[\left(f-\frac{\Gamma_{1}+\gamma_{1}}{2}\right)^{2}\right]=\frac{1}{\int_{a}^{b} h(t) d t} \int_{a}^{b}\left(f-\frac{\Gamma_{1}+\gamma_{1}}{2}\right)^{2} h(t) d t \\
& \leq \frac{1}{\int_{a}^{b} h(t) d t} \int_{a}^{b}\left(\frac{\Gamma_{1}-\gamma_{1}}{2}\right)^{2} h(t) d t=\frac{\left(\Gamma_{1}-\gamma_{1}\right)^{2}}{4} .
\end{aligned}
$$

Lemma 2 Let $f$ be a Riemann-integrable function defined on $[a, b]$ with $\gamma_{1} \leq f(x) \leq \Gamma_{1}$, where $\gamma_{1}$ and $\Gamma_{1}$ are two constants and a Riemann-integrable function $h:[a, b] \rightarrow[0, \infty)$ with $\int_{a}^{b} h(x) d x>0$. Then we have the following relations:

$$
\operatorname{var}_{h}(f) \leq\left(\Gamma_{1}-M_{h}[f]\right)\left(M_{h}[f]-\gamma_{1}\right)
$$

or

$$
\operatorname{var}_{h}(f) \leq\left(\Gamma_{1}-\frac{\int_{a}^{b} f(x) h(x) d x}{\int_{a}^{b} h(x) d x}\right)\left(\frac{\int_{a}^{b} f(x) h(x) d x}{\int_{a}^{b} h(x) d x}-\gamma_{1}\right)
$$

Proof It is easy to see that $\operatorname{var}_{h}(f)=\frac{1}{\int_{a}^{b} h(x) d x} \int_{a}^{b}\left(f(x)-\frac{\int_{a}^{b} f(t) h(t) d t}{\int_{a}^{b} h(t) d t}\right)^{2} h(x) d x$ can be rewritten thus:

$$
\operatorname{var}_{h}(f)=\frac{\int_{a}^{b} f^{2}(x) h(x) d x}{\int_{a}^{b} h(x) d x}-\left[\frac{\int_{a}^{b} f(x) h(x) d x}{\int_{a}^{b} h(x) d x}\right]^{2} .
$$

Next, using the idea of Dragomir [6], we will make several calculations, namely

$$
\begin{aligned}
& \frac{\int_{a}^{b}\left(\Gamma_{1}-f(x)\right)\left(f(x)-\gamma_{1}\right) h(x) d x}{\int_{a}^{b} h(x) d x} \\
& =-\Gamma_{1} \gamma_{1}+\left(\Gamma_{1}+\gamma_{1}\right) \frac{\int_{a}^{b} f(x) h(x) d x}{\int_{a}^{b} h(x) d x}-\frac{\int_{a}^{b} f^{2}(x) h(x) d x}{\int_{a}^{b} h(x) d x} \\
& =-\Gamma_{1} \gamma_{1}+\left(\Gamma_{1}+\gamma_{1}\right) \frac{\int_{a}^{b} f(x) h(x) d x}{\int_{a}^{b} h(x) d x}-\operatorname{var}_{h}(f)-\left[\frac{\int_{a}^{b} f(x) h(x) d x}{\int_{a}^{b} h(x) d x}\right]^{2} \\
& =\left(\Gamma_{1}-\frac{\int_{a}^{b} f(x) h(x) d x}{\int_{a}^{b} h(x) d x}\right)\left(\frac{\int_{a}^{b} f(x) h(x) d x}{\int_{a}^{b} h(x) d x}-\gamma_{1}\right)-\operatorname{var}_{h}(f) .
\end{aligned}
$$


Since $f$ is a bounded function defined on $[a, b]$ with $\gamma_{1} \leq f(x) \leq \Gamma_{1}$, and the function $h$ : $[a, b] \rightarrow[0, \infty)$, it follows that $\left(\Gamma_{1}-f(x)\right)\left(f(x)-\gamma_{1}\right) \geq 0$. Therefore, it is easy to see that $\frac{\int_{a}^{b}\left(\Gamma_{1}-f(x)\right)\left(f(x)-\gamma_{1}\right) h(x) d x}{\int_{a}^{b} h(x) d x} \geq 0$. This inequality proved the inequality of the statement.

Next we show several relations between $h$-variance and $h$-covariance.

Lemma 3 If $f, g \in \mathcal{R}([a, b])$, and a Riemann-integrable function $h:[a, b] \rightarrow[0, \infty)$ with $\int_{a}^{b} h(x) d x>0$, then we have the following equality:

$$
\operatorname{var}_{h}(a f+b g)=a^{2} \operatorname{var}_{h}(f)+b^{2} \operatorname{var}_{h}(g)+2 a b \operatorname{cov}_{h}(f, g)
$$

where $a$ and $b$ are real numbers.

Proof From the expression of the variance, we have

$$
\begin{aligned}
\operatorname{var}_{h}(a f+b g)= & M_{h}\left[(a f+b g)^{2}\right]-M_{h}^{2}[a f+b g] \\
= & M_{h}\left[a^{2} f^{2}+2 a b f g+b^{2} g^{2}\right]-\left(a M_{h}[f]+b M_{h}[g]\right)^{2} \\
= & a^{2} M_{h}\left[f^{2}\right]+2 a b M_{h}[f g]+b^{2} M_{h}\left[g^{2}\right]-a^{2} M_{h}^{2}[f] \\
& -2 a b M_{h}[f] M_{h}[g]-b^{2} M_{h}^{2}[g] \\
= & a^{2} \operatorname{var}_{h}(f)+b^{2} \operatorname{var}_{h}(g)+2 a b \operatorname{cov}_{h}(f, g) .
\end{aligned}
$$

Therefore, we deduce relationship (1).

Proposition 4 If $f, g \in \mathcal{R}([a, b])$, then we have the following equality:

$$
\operatorname{var}_{h}(f+g)=\operatorname{var}_{h}(f)+\operatorname{var}_{h}(g)+2 \operatorname{cov}(f, g)
$$

and

$$
\operatorname{var}_{h}(f-g)=\operatorname{var}_{h}(f)+\operatorname{var}_{h}(g)-2 \operatorname{cov}(f, g) .
$$

Proof If we take $a=b=1$ and $a=1, b=-1$ in relation (7), then we obtain equalities (8) and (9).

Remark 1 From relations (8) and (9), we find the parallelogram law in terms of $h$-variance, namely

$$
\operatorname{var}_{h}(f+g)+\operatorname{var}_{h}(f-g)=2 \operatorname{var}_{h}(f)+2 \operatorname{var}_{h}(g) .
$$

Lemma 5 Iff $, g, p, q \in \mathcal{R}([a, b])$, then we have the following equality:

$$
\begin{aligned}
& \operatorname{cov}_{h}(a f+b g, c p+d q) \\
& \quad=a c \operatorname{cov}_{h}(f, p)+a d \operatorname{cov}_{h}(f, q)+b c \operatorname{cov}_{h}(g, p)+b d \operatorname{cov}_{h}(g, q)
\end{aligned}
$$

where $a, b, c$, and $d$ are real numbers. 
Proof From the expression of the covariance, we have

$$
\begin{aligned}
\operatorname{cov}_{h}(a f+b g, c p+d q)= & M_{h}[(a f+b g)(c p+d q)]-M_{h}[a f+b g] M_{h}[c p+d q] \\
= & M_{h}[a c f p+a d f q+b c g p+b d g q] \\
& -\left(a M_{h}[f]+b M_{h}[g]\right)\left(c M_{h}[p]+d M_{h}[q]\right) \\
= & a c\left(M_{h}[f p]-M_{h}[f] M_{h}[p]\right)+a d\left(M_{h}[f q]-M_{h}[f] M_{h}[q]\right) \\
& +b c\left(M_{h}[g p]-M_{h}[g] M_{h}[p]\right)+b d\left(M_{h}[g q]-M_{h}[g] M_{h}[q]\right) \\
= & a c \operatorname{cov}_{h}(f, p)+a d \operatorname{cov}_{h}(f, q)+b c \operatorname{cov}_{h}(g, p)+b d \operatorname{cov}_{h}(g, q) .
\end{aligned}
$$

We can prove an inequality for integrable functions similar to the inequality of CauchySchwarz for random variables given by the following.

Theorem 6 Iff, $g, h \in \mathcal{R}([a, b])$, then we have the inequality

$$
\left|\operatorname{cov}_{h}(f, g)\right|^{2} \leq \operatorname{var}_{h}(f) \operatorname{var}_{h}(g) \quad \text { or }\left|\operatorname{cov}_{h}(f, g)\right| \leq \sqrt{\operatorname{var}_{h}(f) \operatorname{var}_{h}(g)} .
$$

Proof If $\operatorname{var}_{h}(g)=0$, then relation (12) is true. If $\operatorname{var}_{h}(g) \neq 0$, then we calculate the $h$ variance for the function:

$$
w=f-\frac{\operatorname{cov}_{h}(f, g)}{\operatorname{var}_{h}(g)} g \text { thus } \quad \operatorname{var}_{h}(w)=\operatorname{var}_{h}(f)-\frac{\operatorname{cov}_{h}^{2}(f, g)}{\operatorname{var}_{h}(g)} \geq 0,
$$

because $\operatorname{var}_{h}(w) \geq 0$.

Proposition 7 Let $f$ and $g$ be two Riemann-integrable functions defined on $[a, b]$ with $\gamma_{1} \leq f(x) \leq \Gamma_{1}$ and $\gamma_{2} \leq g(x) \leq \Gamma_{2}$, where $\gamma_{1}, \gamma_{2}, \Gamma_{1}, \Gamma_{2}$ are four constants, and we have a Riemann-integrable $h$ function, $h:[a, b] \rightarrow[0, \infty)$ with $\int_{a}^{b} h(x) d x>0$. Then we have

$$
\begin{aligned}
\left|\operatorname{cov}_{h}(f, g)\right| & =|T(f, g ; h)| \\
& \leq \sqrt{\left(\Gamma_{1}-M_{h}[f]\right)\left(M_{h}[f]-\gamma_{1}\right)\left(\Gamma_{2}-M_{h}[g]\right)\left(M_{h}[g]-\gamma_{2}\right)} \\
& \leq \frac{1}{4}\left(\Gamma_{1}-\gamma_{1}\right)\left(\Gamma_{2}-\gamma_{2}\right) .
\end{aligned}
$$

Proof Using Theorem 6 and Lemma 2, we deduce the statement.

Remark 2 Inequality (13) is a refinement of inequality of Grüss, because

$$
\begin{aligned}
\left|\operatorname{cov}_{h}(f, g)\right| & =|T(f, g ; h)|=\left|M_{h}[f g]-M_{h}[f] M_{h}[g]\right| \\
& \leq \sqrt{\left(\Gamma_{1}-M_{h}[f]\right)\left(M_{h}[f]-\gamma_{1}\right)\left(\Gamma_{2}-M_{h}[g]\right)\left(M_{h}[g]-\gamma_{2}\right)} \\
& \leq \frac{1}{4}\left(\Gamma_{1}-\gamma_{1}\right)\left(\Gamma_{2}-\gamma_{2}\right) .
\end{aligned}
$$

Remark 3 In the proof of Lemma 2 we found the equality in terms of random variables, given by

$$
\operatorname{cov}\left(\Gamma_{1}-f, f-\gamma_{1}\right)=\left(\Gamma_{1}-M_{h}[f]\right)\left(M_{h}[f]-\gamma_{1}\right)-\operatorname{var}_{h}(f) .
$$


Remark 4 (a) Let $f$ be a Riemann-integrable function defined on $[a, b]$ with $\gamma_{1} \leq f(x) \leq \Gamma_{1}$, where $\gamma_{1}, \Gamma_{1}$ are two constants. Then we have

$$
\operatorname{var}(f) \leq \frac{1}{4}\left(\Gamma_{1}-\gamma_{1}\right)^{2}
$$

(b) Let $f$ be a Riemann-integrable function defined on $[a, b]$ with $\gamma_{1} \leq f(x) \leq \Gamma_{1}$, where $\gamma_{1}$, $\Gamma_{1}$ are two constants. Then we have

$$
\operatorname{var}(f) \leq\left(\Gamma_{1}-M_{h}[f]\right)\left(M_{h}[f]-\gamma_{1}\right)
$$

or

$$
\operatorname{var}(f) \leq\left(\Gamma_{1}-\frac{1}{b-a} \int_{a}^{b} f(x) d x\right)\left(\frac{1}{b-a} \int_{a}^{b} f(x) d x-\gamma_{1}\right)
$$

(c) Let $f$ and $g$ be two Riemann-integrable functions defined on $[a, b]$ with $\gamma_{1} \leq f(x) \leq \Gamma_{1}$ and $\gamma_{2} \leq g(x) \leq \Gamma_{2}$ where $\gamma_{1}, \gamma_{2}, \Gamma_{1}, \Gamma_{2}$ are four constants. Then we have

$$
\begin{aligned}
& \left|\frac{1}{b-a} \int_{a}^{b} f(x) g(x) d x-\frac{1}{b-a} \int_{a}^{b} f(x) d x \frac{1}{b-a} \int_{a}^{b} g(x) d x\right| \\
& \leq \sqrt{\left(\Gamma_{1}-\frac{1}{b-a} \int_{a}^{b} f(x) d x\right)\left(\frac{1}{b-a} \int_{a}^{b} f(x) d x-\gamma_{1}\right)} \\
& \quad \times \sqrt{\left(\Gamma_{2}-\frac{1}{b-a} \int_{a}^{b} g(x) d x\right)\left(\frac{1}{b-a} \int_{a}^{b} g(x) d x-\gamma_{2}\right)} \\
& \leq \frac{1}{4}\left(\Gamma_{1}-\gamma_{1}\right)\left(\Gamma_{2}-\gamma_{2}\right) .
\end{aligned}
$$

Theorem 8 Iff $, g, q \in \mathcal{R}([a, b])$, with $f \neq k q$ and $\operatorname{var}(f) \neq 0$, then we have the inequality

$$
0 \leq \frac{\left[\operatorname{cov}_{h}(f, g) \operatorname{cov}_{h}(f, q)-\operatorname{cov}_{h}(g, q) \operatorname{var}_{h}(f)\right]^{2}}{\operatorname{var}_{h}(f) \operatorname{var}_{h}(q)-\left[\operatorname{cov}_{h}(f, g)\right]^{2}} \leq \operatorname{var}_{h}(f) \operatorname{var}_{h}(g)-\left[\operatorname{cov}_{h}(f, g)\right]^{2} .
$$

Proof For the integrable functions $f, g$ and $q$, with $\operatorname{var}(f) \neq 0$, we take the following integrable function:

$$
w=\frac{\operatorname{cov}_{h}(f, g)+\lambda \operatorname{cov}_{h}(f, q)}{\operatorname{var}_{h}(f)} f-g-\lambda q .
$$

We calculate the variance of the function $w$, thus:

$$
\operatorname{var}_{h}(w)=\operatorname{var}_{h}\left(\left(\frac{\operatorname{cov}_{h}(f, g)}{\operatorname{var}_{h}(f)} f-g\right)+\lambda\left(\frac{\operatorname{cov}_{h}(f, q)}{\operatorname{var}_{h}(f)} f-q\right)\right)
$$

and applying Lemma 3, we have

$$
\begin{aligned}
\operatorname{var}_{h}(w)= & \operatorname{var}_{h}\left(\frac{\operatorname{cov}_{h}(f, g)}{\operatorname{var}_{h}(f)} f-g\right)+\lambda^{2} \operatorname{var}_{h}\left(\frac{\operatorname{cov}_{h}(f, q)}{\operatorname{var}_{h}(f)} f-q\right) \\
& +2 \lambda \operatorname{cov}_{h}\left(\frac{\operatorname{cov}_{h}(f, g)}{\operatorname{var}_{h}(f)} f-g, \frac{\operatorname{cov}_{h}(f, q)}{\operatorname{var}_{h}(f)} f-q\right)
\end{aligned}
$$




$$
\begin{aligned}
= & \operatorname{var}_{h}(g)-\frac{\left[\operatorname{cov}_{h}(f, g)\right]^{2}}{\operatorname{var}_{h}(f)}+\lambda^{2}\left(\operatorname{var}_{h}(q)-\frac{\left[\operatorname{cov}_{h}(f, q)\right]^{2}}{\operatorname{var}_{h}(f)}\right) \\
& +2 \lambda \operatorname{cov}_{h}\left(\frac{\operatorname{cov}_{h}(f, g)}{\operatorname{var}_{h}(f)} f-g, \frac{\operatorname{cov}_{h}(f, q)}{\operatorname{var}_{h}(f)} f-q\right) .
\end{aligned}
$$

Using Lemma 2, we deduce the following inequality:

$$
\begin{aligned}
\operatorname{cov}_{h}\left(\frac{\operatorname{cov}_{h}(f, g)}{\operatorname{var}_{h}(f)} f-g, \frac{\operatorname{cov}_{h}(f, q)}{\operatorname{var}_{h}(f)} f-q\right) \\
=\frac{\operatorname{cov}_{h}(f, g) \operatorname{cov}_{h}(f, q)}{\operatorname{var}_{h}(f) \operatorname{var}_{h}(f)} \operatorname{cov}_{h}(f, f) \\
\quad-\frac{\operatorname{cov}_{h}(f, g) \operatorname{cov}_{h}(f, q)}{\operatorname{var}_{h}(f)}-\frac{\operatorname{cov}_{h}(f, q) \operatorname{cov}_{h}(f, g)}{\operatorname{var}_{h}(f)}+\operatorname{cov}_{h}(g, q) \\
=\operatorname{cov}_{h}(g, q)-\frac{\operatorname{cov}_{h}(f, g) \operatorname{cov}_{h}(f, q)}{\operatorname{var}_{h}(f)} .
\end{aligned}
$$

Returning to calculation of the function $w$, we have

$$
\begin{aligned}
\operatorname{var}_{h}(w)= & \operatorname{var}_{h}(g)-\frac{\left[\operatorname{cov}_{h}(f, g)\right]^{2}}{\operatorname{var}_{h}(f)}+\lambda^{2}\left(\operatorname{var}_{h}(q)-\frac{\left[\operatorname{cov}_{h}(f, q)\right]^{2}}{\operatorname{var}_{h}(f)}\right) \\
& +2 \lambda\left(\operatorname{cov}_{h}(g, q)-\frac{\operatorname{cov}_{h}(f, g) \operatorname{cov}_{h}(f, q)}{\operatorname{var}_{h}(f)}\right) .
\end{aligned}
$$

Therefore, we deduce the equality

$$
\begin{aligned}
\operatorname{var}_{h}(f) \operatorname{var}_{h}(w)= & \operatorname{var}_{h}(f) \operatorname{var}_{h}(g)-\left[\operatorname{cov}_{h}(f, g)\right]^{2}+\lambda^{2}\left(\operatorname{var}_{h}(f) \operatorname{var}_{h}(q)-\left[\operatorname{cov}_{h}(f, q)\right]^{2}\right) \\
& +2 \lambda\left(\operatorname{var}_{h}(f) \operatorname{cov}_{h}(g, q)-\operatorname{cov}_{h}(f, g) \operatorname{cov}_{h}(f, q)\right) .
\end{aligned}
$$

Since $\operatorname{var}_{h}(f) \operatorname{var}_{h}(w) \geq 0$, it follows that

$$
\begin{aligned}
& \lambda^{2}\left(\operatorname{var}_{h}(f) \operatorname{var}_{h}(q)-\left[\operatorname{cov}_{h}(f, q)\right]^{2}\right)+2 \lambda\left(\operatorname{var}_{h}(f) \operatorname{cov}_{h}(g, q)-\operatorname{cov}_{h}(f, g) \operatorname{cov}_{h}(f, q)\right) \\
& \quad+\operatorname{var}_{h}(f) \operatorname{var}_{h}(g)-\left[\operatorname{cov}_{h}(f, g)\right]^{2} \geq 0
\end{aligned}
$$

for every $\lambda \in \mathbf{R}$.

This implies that

$$
\begin{aligned}
& \left(\operatorname{var}_{h}(f) \operatorname{var}_{h}(q)-\left[\operatorname{cov}_{h}(f, q)\right]^{2}\right)\left(\operatorname{var}_{h}(f) \operatorname{var}_{h}(g)-\left[\operatorname{cov}_{h}(f, g)\right]^{2}\right) \\
& \quad \geq\left(\operatorname{var}_{h}(f) \operatorname{cov}_{h}(g, q)-\operatorname{cov}_{h}(f, g) \operatorname{cov}_{h}(f, q)\right)^{2} .
\end{aligned}
$$

Taking into account that $\operatorname{var}_{h}(f) \operatorname{var}_{h}(q)-\left[\operatorname{cov}_{h}(f, q)\right]^{2} \neq 0$, because $f \neq k q$ and dividing by $\operatorname{var}_{h}(f) \operatorname{var}_{h}(q)-\left[\operatorname{cov}_{h}(f, q)\right]^{2}$, we obtain the inequality of the statement.

Remark 5 Let $f, g$ and $q$ be three integrable functions, with $\operatorname{var}_{h}(g) \neq 0$ and $\operatorname{var}_{h}(q) \neq 0$. If we take the following function:

$$
w=f-\frac{\operatorname{cov}_{h}(f, g)}{\operatorname{var}_{h}(g)} g-\lambda q,
$$


then we have the inequality

$$
\begin{aligned}
0 & \leq \frac{\left[\operatorname{cov}_{h}(f, q) \operatorname{cov}_{h}(g, q)-\operatorname{cov}_{h}(f, q) \operatorname{var}_{h}(g)\right]^{2}}{\operatorname{var}_{h}(g) \operatorname{var}_{h}(q)} \\
& \leq \operatorname{var}_{h}(f) \operatorname{var}_{h}(g)-\left|\operatorname{cov}_{h}(f, g)\right|^{2} .
\end{aligned}
$$

Lemma 9 Let $f$, $g$ be two Riemann-integrable functions defined on $[a, b]$. Then we have

$$
M_{h}\left[f^{2}\right] M_{h}\left[g^{2}\right] \geq M_{h}^{2}[f g]
$$

Proof If $M_{h}\left[f^{2}\right]=0$, then relation (21) is true. Now we consider that $M_{h}\left[f^{2}\right] \neq 0$.

$$
M_{h}\left[(\lambda f-g)^{2}\right] \geq 0,
$$

for all $\lambda \in \mathbf{R}$, so, we have

$$
M_{h}\left[(\lambda f-g)^{2}\right]=\lambda^{2} M_{h}\left[f^{2}\right]-2 \lambda M_{h}[f g]+M_{h}\left[g^{2}\right],
$$

which means that

$$
\lambda^{2} M_{h}\left[f^{2}\right]-2 \lambda M_{h}[f g]+M_{h}\left[f^{2}\right] \geq 0,
$$

for all $\lambda \in \mathbf{R}$, which implies

$$
M_{h}\left[f^{2}\right] M_{h}\left[g^{2}\right] \geq M_{h}^{2}[f g]
$$

Lemma 9 represents generalized integral variant of inequality of Cauchy.

Now we compare inequalities (21) and (12) to see which is stronger.

Theorem 10 Let $f, g$ be two Riemann-integrable functions defined on $[a, b]$. Then we have

$$
M_{h}\left[f^{2}\right] M_{h}\left[g^{2}\right]-M_{h}^{2}[f g] \geq\left[\operatorname{var}_{h}(f) \operatorname{var}_{h}(g)-\operatorname{cov}_{h}^{2}(f, g)\right] \geq 0 .
$$

Proof We calculate the difference of the terms which appear in inequality (12) and (21), thus:

$$
\begin{aligned}
& M_{h}\left[f^{2}\right] M_{h}\left[g^{2}\right]-M_{h}^{2}[f g]-\left[\operatorname{var}_{h}(f) \operatorname{var}_{h}(g)-\operatorname{cov}_{h}^{2}(f, g)\right] \\
& \quad=M_{h}\left[f^{2}\right] M_{h}^{2}[g]-2 M_{h}[f g] M_{h}[f] M_{h}[g]+M_{h}\left[g^{2}\right] M_{h}^{2}[f] .
\end{aligned}
$$

But, applying the inequality between the arithmetic mean and the geometric mean and Lemma 9, we deduce the relation

$$
\begin{aligned}
M_{h}\left[f^{2}\right] M_{h}^{2}[g]+M_{h}\left[g^{2}\right] M_{h}^{2}[f] & \geq 2 \sqrt{M_{h}\left[f^{2}\right] M_{h}\left[g^{2}\right]} M_{h}[f] M_{h}[g] \\
& \geq 2 M_{h}[f g] M_{h}[f] M_{h}[g] .
\end{aligned}
$$


From this, we obtain the inequality

$$
M_{h}\left[f^{2}\right] M_{h}^{2}[g]-2 M_{h}[f g] M_{h}[f] M_{h}[g]+M_{h}\left[g^{2}\right] M_{h}^{2}[f] \geq 0 .
$$

Consequently the statement is true.

\section{A refinement of Grüss' inequality for normalized isotonic linear functionals}

There are many directions in which the inequality of Grüss [1] has been generalized.

Using the notion of normalized isotonic linear functional which appears in the paper [22], we will give a generalization of inequality of Grüss which is related to a theorem of Andrica and Badea (1988), [23].

Let $E$ be a nonempty set, $L$ a linear class of real-valued functions and $g: E \rightarrow \mathbf{R}$ having the properties:

(L1) $f, g \in L$ imply $(\alpha f+\beta g) \in L$ for all $\alpha, \beta \in \mathbf{R}$,

(L2) $\mathbf{1} \in L$, i.e. if $f_{0}(t)=1,(\forall) t \in E$, then $f_{0} \in L$.

An isotonic linear functional (in [24] is called positive definite functional) $A: L \rightarrow \mathbf{R}$ is a functional satisfying:

(A1) $A(\alpha f+\beta g)=\alpha A(f)+\beta A(g)$, for all $f, g \in L$ and $\alpha, \beta \in \mathbf{R}$.

(A2) If $f \in L$ and $f \geq 0$, then $A(f) \geq 0$.

(A3) The mapping $A$ is said to be normalized if $A(\mathbf{1})=1$.

Theorem 11 Let $f \in L$ be such that $f^{2} \in L$ and assume that there exist real numbers $\gamma_{1}$ and $\Gamma_{1}$ so that $\gamma_{1} \leq f \leq \Gamma_{1}$.

Then for any normalized isotonic linear functional $A: L \rightarrow \mathbf{R}$ one has the inequality

$$
A\left(f^{2}\right)-[A(f)]^{2} \leq\left(\Gamma_{1}-A(f)\right)\left(A(f)-\gamma_{1}\right) .
$$

Proof Taking into account the hypothesis we have by using (L1) and (L2) that $\left(f-\gamma_{1} \cdot \mathbf{1}\right)\left(\Gamma_{1}\right.$. $\mathbf{1}-f) \geq 0$ and thus by (A2), we see that

$$
A\left[\left(f-\gamma_{1} \cdot \mathbf{1}\right)\left(\Gamma_{1} \cdot \mathbf{1}-f\right)\right] \geq 0 .
$$

Using also (A1) and (A3) we have,

$$
A\left[f \Gamma_{1} \cdot \mathbf{1}-\gamma_{1} \Gamma_{1} \cdot \mathbf{1}-f^{2}+\gamma_{1} f \cdot \mathbf{1}\right] \geq 0
$$

or

$$
A(f) \Gamma_{1}-\gamma_{1} \Gamma_{1} \cdot A(\mathbf{1})-A\left(f^{2}\right)+\gamma_{1} A(f) \geq 0
$$

or

$$
\left(\Gamma_{1}+\gamma_{1}\right) A(f)-\gamma_{1} \Gamma_{1} \cdot A(\mathbf{1}) \geq A\left(f^{2}\right) .
$$

Therefore

$$
\left(\Gamma_{1}+\gamma_{1}\right) A(f)-\gamma_{1} \Gamma_{1} \cdot A(\mathbf{1})-[A(f)]^{2} \geq A\left(f^{2}\right)-[A(f)]^{2}
$$


and from this we obtain

$$
\left(\Gamma_{1}-A(f)\right)\left(A(f)-\gamma_{1}\right) \geq A\left(f^{2}\right)-[A(f)]^{2} .
$$

From the inequality of Cauchy-Schwarz for a normalized isotonic linear functional, [22], a counterpart of the CBS inequality, we obtain for $f, g, f^{2}, g^{2} \in L$ where $f, g: E \rightarrow \mathbf{R}$ and $A: L \rightarrow \mathbf{R}$ is any normalized isotonic linear functional

$$
[A(f g)]^{2} \leq A\left(f^{2}\right) A\left(g^{2}\right)
$$

Now if we consider this inequality and previous theorem we deduce as in Lemma 2 the following result.

Theorem 12 Let $f, g, f g \in L$ such that $f^{2}, g^{2} \in L$ and $\gamma_{1} \leq f \leq \Gamma_{1}, \gamma_{2} \leq g \leq \Gamma_{2}$ where $\gamma_{1}, \gamma_{2}$, $\Gamma_{1}, \Gamma_{2}$ are given real numbers. Then for any normalized linear isotonic functional $A: L \rightarrow \mathbf{R}$ one has the inequality

$$
A(f g)-A(f) A(g) \leq \sqrt{\left(\Gamma_{1}-A(f)\right)\left(A(f)-\gamma_{1}\right)\left(\Gamma_{2}-A(g)\right)\left(A(g)-\gamma_{2}\right)} .
$$

Proof Because we can write

$$
\begin{aligned}
A[(f-A(f) \cdot \mathbf{1})(g-A(g) \cdot \mathbf{1})] & =A[(f-A(f) \cdot \mathbf{1}) g]-A[(f-A(f) \cdot \mathbf{1}) A(g)] \\
& =A(f g)-A(f) A(g)
\end{aligned}
$$

we can apply the CBS-inequality for a normalized linear isotonic functional, if $(f-A(f)$. $\mathbf{1})^{2},(g-A(g) \cdot \mathbf{1})^{2},(f-A(f) \cdot \mathbf{1})(g-A(g) \cdot \mathbf{1}) \in L$, by Theorem 11 we have

$$
\begin{aligned}
A(f g)-A(f) A(g) & =A[(f-A(f) \cdot \mathbf{1})(g-A(g) \cdot \mathbf{1})] \\
& \leq \sqrt{A\left((f-A(f) \cdot \mathbf{1})^{2}\right) A\left((g-A(g) \cdot \mathbf{1})^{2}\right)} \\
& \leq \sqrt{\left(\Gamma_{1}-A(f)\right)\left(A(f)-\gamma_{1}\right)\left(\Gamma_{2}-A(g)\right)\left(A(g)-\gamma_{2}\right)} .
\end{aligned}
$$

Remark 6 If we take in the first theorem $A=\frac{1}{b-a} \int_{a}^{b}, L=L(a, b)$ (the Lebesque space of integrable functions on $[a, b]$ ) and $g$ satisfying the condition $\gamma_{2} \leq g \leq \Gamma_{2}$ on the interval $[a, b]$, then we obtain inequality (17).

Theorem 13 Let $f, g, h \in L$ be such that $h \geq 0, f h, g h, f g h \in L$ and there exist real constants $\gamma_{2}, \Gamma_{2}$ so that $\gamma_{2} \leq g \leq \Gamma_{2}$. Then for any $B: L \rightarrow \mathbf{R}$ an isotonic linear functional so that $B(h)>0$ one has the inequality

$$
\begin{aligned}
& \left|\frac{B(f g h)}{B(h)}-\frac{B(f h)}{B(h)} \frac{B(g h)}{B(h)}\right| \\
& \quad \leq \sqrt{\left(\Gamma_{1}-\frac{B(f h)}{B(h)}\right)\left(\frac{B(f h)}{B(h)}-\gamma_{1}\right)\left(\Gamma_{2}-\frac{B(g h)}{B(h)}\right)\left(\frac{B(g h)}{B(h)}-\gamma_{2}\right)} .
\end{aligned}
$$


Proof We use the normalized isotonic linear functional $A$ on $L$ defined by

$$
A_{h}(f)=\frac{1}{B(h)} B(h f),
$$

$A: L \rightarrow \mathbf{R}$.

\section{Properties of $h$-variance}

1. If $f, g \in \mathcal{R}([a, b])$, then we have the following inequality:

$$
\sqrt{\operatorname{var}_{h}(f+g)} \leq \sqrt{\operatorname{var}_{h}(f)}+\sqrt{\operatorname{var}_{h}(g)} .
$$

Proof From equality (8), we have

$$
\begin{aligned}
\operatorname{var}_{h}(f+g) & =\operatorname{var}_{h}(f)+\operatorname{var}_{h}(g)+2 \operatorname{cov}_{h}(f, g) \\
& =\left(\sqrt{\operatorname{var}_{h}(f)}+\sqrt{\operatorname{var}_{h}(g)}\right)^{2}-2\left(\sqrt{\operatorname{var}_{h}(f) \operatorname{var}_{h}(g)}-\operatorname{cov}_{h}(f, g)\right) .
\end{aligned}
$$

Applying the inequality of Cauchy-Schwarz for integrable functions given by

$$
\left|\operatorname{cov}_{h}(f, g)\right| \leq \sqrt{\operatorname{var}_{h}(f) \operatorname{var}_{h}(g)}
$$

it follows that

$$
\operatorname{var}_{h}(f+g) \leq\left(\sqrt{\operatorname{var}_{h}(f)}+\sqrt{\operatorname{var}_{h}(g)}\right)^{2}
$$

which implies the inequality of the statement.

2. If $f, g \in \mathcal{R}([a, b])$, then we have the following inequality:

$$
\sqrt{\operatorname{var}_{h}(f-g)} \geq\left|\sqrt{\operatorname{var}_{h}(f)}-\sqrt{\operatorname{var}_{h}(g)}\right| .
$$

Proof From relation (9), we have

$$
\begin{aligned}
\operatorname{var}_{h}(f-g) & =\operatorname{var}_{h}(f)+\operatorname{var}_{h}(g)-2 \operatorname{cov}_{h}(f, g) \\
& =\left(\sqrt{\operatorname{var}_{h}(f)}-\sqrt{\operatorname{var}_{h}(g)}\right)^{2}+2\left(\sqrt{\operatorname{var}_{h}(f) \operatorname{var}_{h}(g)}-\operatorname{cov}_{h}(f, g)\right) .
\end{aligned}
$$

Applying the inequality of Cauchy-Schwarz for integrable functions, we obtain

$$
\operatorname{var}_{h}(f-g) \geq\left(\sqrt{\operatorname{var}_{h}(f)}-\sqrt{\operatorname{var}_{h}(g)}\right)^{2},
$$

which implies the inequality of the statement.

3. The natural way to obtain these quantities is by introducing and using the standard inner product (also known as the dot product) on $C^{0}([a, b])$. The inner product of any two continuous $f$ and $g$ functions is defined by

$$
\langle f, g\rangle_{h}=\operatorname{cov}_{h}(f, g)
$$


The result is always a real number. Therefore the set $C^{0}([a, b])$ can be organized as an Euclidean space.

The inner product of $C^{0}([a, b])$ with itself is always non-negative. This product allows us to define the 'length' of an integrable function $f$ through

$$
\|f\|_{h}=\sqrt{\langle f, f\rangle_{h}}=\sqrt{\operatorname{cov}_{h}(f, f)}=\sqrt{\operatorname{var}_{h}(f)} .
$$

This length function satisfies the required properties of a norm and is called the Euclidean norm on $C^{0}([a, b])$.

Finally, one can use the norm to define a metric (or distance function) on $C^{0}([a, b])$ by

$$
d_{h}(f, g)=\|f-g\|_{h}=\sqrt{\operatorname{var}_{h}(f-g)} .
$$

This distance function is called the Euclidean metric. This formula expresses a special case of the Pythagorean theorem.

From relation (27), we have

$$
\sqrt{\operatorname{var}_{h}(f-q)}=\sqrt{\operatorname{var}_{h}((f-g)+(g-q))} \leq \sqrt{\operatorname{var}_{h}(f-g)}+\sqrt{\operatorname{var}_{h}(g-q)}
$$

so we obtain the triangle inequality,

$$
d_{h}(f, q) \leq d_{h}(f, g)+d_{h}(g, q)
$$

Remark 7 From [25], the analog of the arithmetic mean in the context of finite measure spaces $(X, \Sigma ; \mu)$ is the integral arithmetic mean, which, for a $\mu$-integrable function $f: X \rightarrow$ $\mathbf{R}$ is the number

$$
M_{1}(f ; \mu)=\frac{1}{\mu(X)} \int_{X} f d \mu,
$$

where $M_{1}(f ; \mu)$ represents in probability theory the conditional expectation of the random variable $f$.

A result which represents another estimate of Jensen's inequality, in the sense of generalizations of the integral arithmetic mean and variance, can also be found in [25], page 53.

Lemma 14 ([25], 1.8) (Another estimate of Jensen's inequality) Let $(X, \Sigma ; \mu)$ be a finite measure space and let $g: X \rightarrow \mathbf{R}$ be a $\mu$-integrable function (or $g \in L^{2}(\mu)$ ). Iff is a twice differentiable function given on an I interval that includes the image of $g$; and $m \leq f^{\prime \prime} \leq M$, then

$$
\frac{m}{2} \operatorname{var}(g) \leq M_{1}(f \circ g ; \mu)-f\left(M_{1}(g ; \mu)\right) \leq \frac{M}{2} \operatorname{var}(g),
$$

where $\operatorname{var}(g)=M_{1}\left(\left(g-M_{1}(g ; \mu)\right)^{2} ; \mu\right)$ denotes the variance of $g$.

Izumio et al. showed in [26] some extensions of Grüss' inequality and they also studied the integral-type Grüss' inequalities for Lebesque space $L_{\mu}(\Omega)$ for a finite positive measure $\mu$ on $\Omega$. 


\section{Applications}

1. Taking into account the integral arithmetic mean and h-integral arithmetic mean for a Riemann-integrable function $f:[a, b] \rightarrow \mathbf{R}$ we can rewrite the following inequalities.

(a) In the case when $p \geq 0$ the integral form of the inequality from Theorem 2.4 (see [27]) was given by Theorem 2.5 . Under the conditions of Theorem 2.5 , the inequality becomes

$$
M_{1}\left[\frac{f^{m+1}}{g^{p}}\right] \geq \frac{M_{1}^{m+1}[f]}{M_{1}^{p}[g]} .
$$

(b) In [28], Mortici gave a new refinement of Radon's inequality. Using the integral form of the reverse of inequality from Theorem 2.5 (see [27]) we obtain, for $p \in(-1,0), m \in$ $(-1,0)$ and $m \leq p$, if $f, g:[a, b] \rightarrow \mathbf{R}_{+}$are two integrable functions on $[a, b]$ with $g(x)>0$, $(\forall) x \in[a, b]$ a continuous function on $[a, b]$, the inequality

$$
M_{1}\left[\frac{f^{m+1}}{g^{p}}\right] \leq \frac{M_{1}^{m+1}[f]}{M_{1}^{p}[g]} .
$$

(c) From the integral form of the inequality from Consequence 1 (see [29]) we deduce if $f, g:[a, b] \rightarrow \mathbf{R}_{+}$are two integrable functions, $g$ a continuous function on $[a, b], g(x)>0$, $(\forall) x \in[a, b]$ and $f(x) \in(0,1),(\forall) x \in[a, b]$ the following inequality:

$$
M_{1}\left[\frac{f}{g^{r}(1-f)}\right] \geq \frac{1}{M_{1}^{r}[g]} \frac{M_{1}[f]}{1-M_{1}[f]},
$$

where $r \in[0,1)$.

2. (a) Starting from Theorem 2.3, the inequalities (2.5) and (2.6), Theorem 2.7 and Theorem 2.9, given in [30], by using Theorem 12 (see [31]) and Theorem 8 (see [32]) we can obtain the next properties for certain Riemann-integrable functions. If $g(x)>0$ and if $f, g:[a, b] \rightarrow \mathbf{R}_{+}$are two integrable functions on $[a, b]$ with $m \leq \frac{f(x)}{g(x)} \leq M,(\forall) x \in[a, b]$ and $M<1$, then

$$
\begin{aligned}
0 & \leq M_{1}\left[\frac{f g}{g-f}\right]-\frac{M_{1}^{2}[g]}{M_{1}[g]-M_{1}[f]} \\
& \leq M_{1}\left[\frac{f g^{2}}{(g-f)^{2}}\right]-\frac{M_{1}[f] M_{1}^{2}[g]}{M_{1}^{2}[g]-M_{1}^{2}[f]}-\frac{M_{1}[f]}{M_{1}[g]} \cdot\left(M_{1}\left[\frac{g^{3}}{(g-f)^{2}}\right]-\frac{M_{1}^{3}[g]}{\left(M_{1}[g]-M_{1}[f]\right)^{2}}\right)
\end{aligned}
$$

and

$$
\begin{aligned}
0 & \leq M_{1}\left[\frac{f g}{g-f}\right]-\frac{M_{1}^{2}[g]}{M_{1}[g]-M_{1}[f]} \\
& \leq \frac{1}{4}(M-m)\left(\frac{1}{(1-M)^{2}}-\frac{1}{(1-m)^{2}}\right) M_{1}[g] .
\end{aligned}
$$

(b) If $g(x)>0$ and if $f, g:[a, b] \rightarrow \mathbf{R}_{+}$are two integrable functions on $[a, b]$ with $m \leq$ $\frac{f(x)}{g(x)} \leq M,(\forall) x \in[a, b]$ and $M<1$, then we have

$$
0 \leq M_{1}\left[\frac{f g}{g-f}\right]-\frac{M_{1}[f] M_{1}[g]}{M_{1}[f-g]} \leq\left(\frac{M}{1-M}+\frac{m}{1-m}-\frac{2(M+m)}{2-M+m}\right) M_{1}[g]
$$


and

$$
\begin{aligned}
0 & \leq M_{1}\left[\frac{f g}{g-f}\right]-\frac{M_{1}[f] M_{1}[g]}{M_{1}[f-g]} \\
& \leq M_{1}[g] \frac{(M+m) M_{1}[g]-M_{1}[f]}{M_{1}[f+g]-(M+m) M_{1}[g]}-2 \frac{M+m}{2-(M+m)} M_{1}[g]+M_{1}\left[\frac{f g}{g-f}\right] .
\end{aligned}
$$

(c) Using the integral form of the inequality (2.5) and (2.6) from Theorem 2.3 (see [30]), under the conditions of Theorem 5 (see [33]) we find that, for every $n \geq 2, p \geq 1, g(x)>0$ and if $f, g:[a, b] \rightarrow \mathbf{R}_{+}$are two continuous functions on $[a, b]$ with $m=\inf _{[a, b]} \frac{f(x)}{g(x)}, M=$ $\sup _{[a, b]} \frac{f(x)}{g(x)}$, we have

$$
0 \leq M_{1}\left[\frac{f^{p}}{g^{p-1}}\right]-\frac{M_{1}^{p}[f]}{M_{1}^{p-1}[g]} \leq \frac{p}{4}(M-m)\left(M^{p-1}-m^{p-1}\right) M_{1}[g]
$$

If $f, g:[a, b] \rightarrow \mathbf{R}_{+}$are two integrable functions on $[a, b]$ then

$$
\begin{aligned}
0 & \leq M_{1}\left[\frac{f^{p}}{g^{p-1}}\right]-\frac{M_{1}^{p}[f]}{M_{1}^{p-1}[g]} \\
& \leq p\left(M_{1}\left[\frac{f^{p}}{g^{p-1}}\right]-\frac{M_{1}^{p}[f]}{M_{1}^{p-1}[g]}-\frac{M_{1}[f]}{M_{1}[g]}\left(M_{1}\left[\frac{f^{p-1}}{g^{p-2}}\right]-\frac{M_{1}^{p-1}[f]}{M_{1}^{p-2}[g]}\right)\right) .
\end{aligned}
$$

(d) Now we will rewrite the integral form of the inequality (2.19) from Theorem 2.9 (see [30]) which is given in Theorem 6 (see [33]). If $p \geq 1, f$ and $g$ are two continuous functions $f, g:[a, b] \rightarrow \mathbf{R}_{+}$on $[a, b]$, with $m=\inf _{[a, b]} \frac{f(x)}{g(x)}, M=\sup _{[a, b]} \frac{f(x)}{g(x)}$, then we have

$$
\begin{aligned}
0 & \leq M_{1}\left[\frac{f^{p}}{g^{p-1}}\right]-\frac{M_{1}^{p}[f]}{M_{1}^{p-1}[q]} \\
& \leq \frac{\left[(M+m) M_{1}[g]-M_{1}[f]\right]^{p}}{M_{1}^{p-1}[f]}-\frac{(M+m)^{p}}{2^{p-1}} M_{1}[g]+M_{1}\left[\frac{f^{p}}{g^{p-1}}\right] .
\end{aligned}
$$

3. (i) Under the previous conditions we have

$$
0 \leq M_{1}\left[\frac{f^{p}}{g^{p-1}}\right]-\frac{M_{1}^{p}[f]}{M_{1}^{p-1}[q]} \leq\left[M^{p}+m^{p}-\frac{(M+m)^{p}}{2^{p-1}}\right] M_{1}[g] .
$$

(ii) If $f, g:[a, b] \rightarrow \mathbf{R}_{+}$are two integrable functions on $[a, b]$ with $g(x)>0,(\forall) x \in[a, b]$, $p>1$ and $m g(x) \leq f(x) \leq M g(x),(\forall) x \in[a, b]$, then we have

$$
\begin{aligned}
p(p-1) m^{p-2}\left(M_{1}\left[\frac{f^{2}}{g}\right]-\frac{M_{1}^{2}[f]}{M_{1}[g]}\right) & \leq M_{1}\left[\frac{f^{p}}{g^{p-1}}\right]-\frac{M_{1}^{p}[f]}{M_{1}^{p-1}[g]} \\
& \leq p(p-1) M^{p-2}\left(M_{1}\left[\frac{f^{2}}{g}\right]-\frac{M_{1}^{2}[f]}{M_{1}[g]}\right) .
\end{aligned}
$$

4. The following two inequalities are rewritten here and have as a starting point an inequality from [34]. 
(i) If $a, b \in \mathbf{R}, a<b, f:[a, b] \rightarrow \mathbf{R}_{+}$is a convex and continuous function and $h, l:[a, b] \rightarrow$ $\mathbf{R}_{+}$are two integrable functions on $[a, b]$, and, in addition, if $g:[a, b] \rightarrow \mathbf{R}_{+}$is integrable on $[a, b]$, then

$$
\begin{aligned}
& \sup _{x \in[a, b]}\left\{\frac{h(x)}{l(x)}\right\}\left[M_{1}[l \cdot(f \circ g)]-f\left(M_{l}[g]\right) \cdot M_{1}[l]\right] \\
& \geq\left[M_{1}[h \cdot(f \circ g)]-f\left(M_{h}[g]\right) \cdot M_{1}[h]\right] \\
& \geq \inf _{x \in[a, b]}\left\{\frac{h(x)}{l(x)}\right\}\left[M_{1}[l \cdot(f \circ g)]-f\left(M_{l}[g]\right) \cdot M_{1}[l]\right],
\end{aligned}
$$

or

$$
\begin{aligned}
& \sup _{x \in[a, b]}\left\{\frac{h(x)}{l(x)}\right\} M_{1}[l]\left[M_{l}[f \circ g]-f\left(M_{l}[g]\right)\right] \\
& \geq M_{1}[h]\left[M_{h}[f \circ g]-f\left(M_{h}[g]\right)\right] \\
& \geq \inf _{x \in[a, b]}\left\{\frac{h(x)}{l(x)}\right\} M_{1}[l]\left[M_{l}[f \circ g]-f\left(M_{l}[g]\right)\right] .
\end{aligned}
$$

(ii) If $a, b \in \mathbf{R}, a<b, f:[a, b] \rightarrow \mathbf{R}_{+}$is a convex and continuous function, $p, q_{1}:[a, b] \rightarrow$ $\mathbf{R}_{+}$are two integrable functions on $[a, b]$ with $q \in(0,1)$, if $g:[a, b] \rightarrow \mathbf{R}_{+}$is integrable on $[a, b]$ and $M \geq m \geq 0$ are such that $M p(x) \geq q_{1}(x) \geq m p(x),(\forall) x \in \mathbf{R}$, then

$$
\begin{aligned}
M^{\frac{1}{q}}\left(\frac{M_{1}[p]}{M_{1}\left[q_{1}\right]}\right)^{\frac{1}{q}}\left[M_{p}[f \circ g]-f\left(M_{p}[g]\right)\right] & \geq M_{q_{1}}[f \circ g]-f\left(M_{q_{1}}[g]\right) \\
& \geq m^{\frac{1}{q}}\left(\frac{M_{1}[p]}{M_{1}\left[q_{1}\right]}\right)^{\frac{1}{q}}\left[M_{p}[f \circ g]-f\left(M_{p}[g]\right)\right] .
\end{aligned}
$$

5. Starting from the inequality of Halliwell and Mercer (see [35]), we can establish the following result.

If $a, b \in \mathbf{R}, a<b, f, g:[a, b] \rightarrow \mathbf{R}_{+}$are two continuous and strict positive functions on $[a, b]$ and $M_{1}[f]=M_{1}[g]$, then the following inequality holds:

$$
\begin{aligned}
M\left[\frac{g(g-f)^{2}}{g^{2}+\left(\max \left\{\sup _{x \in[a, b]} f, \sup _{x \in[a, b]} g\right\}\right)^{2}}\right] & \leq M\left[f \ln \left(\frac{f}{g}\right)\right] \\
& \leq M\left[\frac{g(g-f)^{2}}{g^{2}+\left(\min \left\{\inf _{x \in[a, b]} f, \inf _{x \in[a, b]} g\right\}\right)^{2}}\right] .
\end{aligned}
$$

\section{Competing interests}

The authors declare that they have no competing interests.

\section{Authors' contributions}

The work presented here was carried out in collaboration between all authors. The study was initiated by NM. The author NM also played the role of the corresponding author. All authors contributed equally and significantly in writing this article. All authors have contributed to, seen and approved the manuscript.

\section{Author details}

'Transilvania University of Brasov, Iuliu Maniu 50, Brasov, 500091, Romania. "Department of Mathematics, "Politehnica"

University of Timisoara, P-ta. Victoriei 2, Timisoara, 300006, Romania. 


\section{Acknowledgements}

We would like to thank the anonymous reviewer for providing valuable comments to improve the manuscript.

\section{Received: 6 November 2013 Accepted: 28 February 2014 Published: 20 Mar 2014}

\section{References}

1. Grüss, G: Uber das Maximum des absoluten Betrages von $\frac{1}{b-a} \int_{a}^{b} f(x) g(x) d x-\frac{1}{b-a} \int_{a}^{b} f(x) d x \frac{1}{b-a} \int_{a}^{b} g(x) d x$. Math. Z. 39 , 215-226 (1935)

2. Acu, A, Gonska, H, Raşa, I: Grüss-type and Ostrowski-type inequalities in approximation theory. Ukr. Math. J. 63, 843-864 (2011)

3. Mitrinović, DS, Pečarić, J, Fink, AM: Classical and New Inequalities in Analysis. Kluwer Academic, Dordrecht (1992)

4. Rusu, MD: On Grüss-type inequalities for positive linear operators. Stud. Univ. Babeş-Bolyai, Math. 56, 551-565 (2011)

5. Peng, G-H, Miao, Y: A note on Grüss type inequality. Appl. Math. Sci. 3(8), 399-402 (2009)

6. Dragomir, SS: Some integral inequalities of Grüss type. Indian J. Pure Appl. Math. 31(4), 397-415 (2000)

7. Dragomir, SS: Some Grüss type inequalities in inner product spaces. JIPAM. J. Inequal. Pure Appl. Math. 4(2), Article ID 42 (2003)

8. Mercer, AMcD: An improvement of the Grüss inequality. JIPAM. J. Inequal. Pure Appl. Math. 6(4), Article ID 93 (2005)

9. Liu, Z: Some sharp Ostrowski-Grüss type inequalities. Univ. Beograd. Publ. Electrotehn. Fak. Ser. Mat. 18, 14-21 (2006)

10. Kechriniotis, A, Delibasis, K: On generalizations of Grüss inequality in inner product spaces and applications. J. Inequal. Appl. 2010, Article ID 167091 (2010)

11. Dahmani, Z, Tabharit, L, Taf, S: New generalizations of Grüss inequality using Riemann-Liouville fractional integrals. Bull. Math. Anal. Appl. 2(3), 93-99 (2010)

12. Cerone, P, Dragomir, SS: Mathematical Inequalities: A Perspective. CRC Press, Taylor \& Francis, New York (2011)

13. Florea, A, Niculescu, CP: A note on Ostrowski's inequality. J. Inequal. Appl. 2005(5), 459-468 (2005)

14. Aldaz, JM: A refinement of the inequality between arithmetic and geometric means. J. Math. Inequal. 2(4), 473-477 (2008)

15. Halmos, P: Finite Dimensional Vector Spaces. Springer, Berlin (1974)

16. Elezović, N, Marangunić, Lj, Pečarić, J: Some improvements of Grüss type inequality. J. Math. Inequal. 1(3), 425-436 (2007)

17. Mitrinović, DS, Pečarić, J: Comments on an inequality of M. Masuyama. SUT J. Math. (Formerly TRU Math.) 27, 89-91 (1991)

18. Pečarić, J: Some further remarks on the Ostrowski generalization of Čebyšev's inequality. J. Math. Anal. Appl. 123, 18-33 (1987)

19. Popoviciu, T: Sur Les Équations Algébriques Ayant Toutes Leurs Racines Réelles. Mathematica 9, 129-145 (1935)

20. Bhatia, R, Davis, C: A better bound on the variance. Am. Math. Mon. 107, 353-357 (2000)

21. Zitikis, R: Grüss's inequality, its probabilistic interpretation, and a sharper bound. J. Math. Inequal. 3(1), 15-20 (2009)

22. Dragomir, SS: A survey of Jessen's type inequalities for positive functionals. RGMIA Res. Rep. Collect. (2011). 46 pp.

23. Andrica, D, Badea, C: Grüss' inequality for positive linear functionals. Period. Math. Hung. 19(2), 155-167 (1998)

24. Pečarić, J, Peric, J: Remarks on the paper "Jensen's inequality and new entropy bounds" of S. Simic. J. Math. Inequal. 6(4), 631-636 (2012)

25. Niculescu, CP, Persson, L-E: Convex Functions and Their Applications. A Contemporary Approach. Springer, Berlin (2006)

26. Izumio, S, Pečarić, J, Tepeš, B: Some extensions of Grüss's inequality. Math. J. Toyama Univ. 26, 61-73 (2003)

27. Batinetu-Giurgiu, DM, Marghidanu, D, Pop, OT: A new generalization of Radon's inequality and applications. Creative Math. Inform. 2, 111-116 (2011)

28. Mortici, C: A new refinement of the Radon inequality. Math. Commun. 16, 319-324 (2011)

29. Ciurdariu, L: Integral forms for some inequalities. Int. J. Math. Anal. 7(53), 2611-2618 (2013)

30. Raţiu, A, Minculete, N: Several refinements and counterparts of Radon's inequality. Math. Bohem. (to appear)

31. Ciurdariu, L: On several inequalities deduced using a power series approach. Int. J. Contemp. Math. Sci. 8(18), 855-864 (2013)

32. Ciurdariu, L: On several inequalities obtained from generalizations of Young's inequality by a power series approach Int. J. Algebra 7(18), 847-856 (2013)

33. Ciurdariu, L: On integral forms of several inequalities. J. Sci. Arts 2(23), 159-166 (2013)

34. Dragomir, SS: Bounds for the normalised Jensen functional. Bull. Aust. Math. Soc. 74, 471-478 (2006)

35. Halliwell, GT, Mercer, PR: A refinement of an inequality from information theory. JPAM. J. Inequal. Pure Appl. Math 5(1), Article ID 3 (2004)

10.1186/1029-242X-2014-119

Cite this article as: Minculete and Ciurdariu: A generalized form of Grüss type inequality and other integral inequalities. Journal of Inequalities and Applications 2014, 2014:119 